\title{
Sintomatología de Salud mental en un hospital público de la Ciudad del Cusco
}

\section{Mental Health symptoms in a public hospital in the City of Cusco}

\author{
Recibido: 11 de Setiembre 2018 | Aceptado: 19 de Noviembre 2018
}

Andy Alvarado ${ }^{1,2}$

\author{
${ }^{1}$ Hospital Regional del Cusco \\ ${ }^{2}$ Centro de Investigación Ambiente, Comportamiento y Sociedad-CIACOMS \\ Correo Electrónico:psiko.andyjoss@gmail.com
}

\begin{abstract}
Resumen
El presente estudio tiene por objetivo observar los niveles de ansiedad, depresión, estrés como principales síntomas de salud mental, además extiende este estudio con los niveles de alcoholismo; estos problemas psicosociales, se abordan en el contexto de un hospital público de la ciudad del Cusco, para determinar la prevalencia de malestar psicológico significativo. Se uso una metodología cuantitativa de nivel descriptivo. Los resultados exponen grandes niveles de ansiedad, y leve presencia de depresión, como también niveles manejables de estrés y niveles no significativos de consumo de alcohol.
\end{abstract}

Palabras Clave: Salud mental, ansiedad, depresión, estrés, alcoholismo, personal sanitario.

\begin{abstract}
The present study aims to observe the levels of anxiety, depression, stress as the main symptoms of mental health, it also extends this study with the levels of alcoholism; These psychosocial problems are addressed in the context of a public hospital in the city of Cusco, to determine the prevalence of significant psychological distress. A descriptive level quantitative methodology was used. The results show high levels of anxiety, and a slight presence of depression, as well as manageable levels of stress and insignificant levels of alcohol consumption.
\end{abstract}

Keywords: Mental health, anxiety, depression, stress, alcoholism, health personnel.

\section{Introducción}

Los cambio políticos, tecnológicos y económicos en los últimos 30 años han repercutido de directamente en los espacios y diseños de trabajo, esto, según GilMonte (2010) ha generado gran impacto en el desempeño laboral e incluso en la calidad 
de vida de los empleados. Una institución hospitalaria es una entidad organizada, atendida, que está dirigida por recurso humano competente y especializado, cuyo principal objetivo es proveer de diversas maneras los medios necesarios para la prevención, diagnostico y tratamiento de enfermedades, dándoles solución, incluso a los aspectos sociales, desde un aspecto médico; en el ámbito hospitalario, es reconocido que los trabajadores entran en contacto con varios riesgos, categorizándose como centros de trabajos con alto riesgo (Nacional Institute of Occupational Safety and Health (NIOSH) de los Estados Unidos de Norteamérica).

El contexto de la salud en los últimos años, ha comprendido una dinámica constante de cambios, motivado por importantes modificaciones del perfil de la población, es decir, los cambios sociales, demográficos y epidemiológicos, acompañado de una vertiginosa innovación tecnológica; dichos cambios e implicaciones en los servicios del complejo sistema sanitario ha planteado grandes desafíos y exigencias al personal trabajador, dado que, la complejidad de las tareas, la actualización de capacidades, el aumento en la demanda de asistencia, ha generado una prolongación en las jornadas de trabajo, que han acompañado a inestabilidad laboral, afectación en ámbitos sociales y familiares, como también, una modificación en el perfil de salud de los propios trabajadores de los establecimientos de salud, donde asumen especial relevancia los problemas psicosociales (Neffa, 2003). Estos factores psicosociales pueden favorecer o perjudicar el trabajo y la calidad de vida del personal de salud, convirtiéndose en alguna clase de riesgo psicosocial (ansiedad, estrés, depresión y consumo de sustancias). Se ha evidenciado, que, el trabajador, tomando en cuenta sus características individuales como personalidad, edad, motivación,experiencia, etc., va asumir dichos riesgos de diversas maneras, por lo que la exposición a estos riesgos no necesariamente afectará negativamente sobre ellos, no obstante, se ha demostrado, que el padecimiento de estos riesgos, se han padecido de manera crónica, y además que la exposición a estos supone una tensión continua, que se manifestará por medio de alteraciones a nivel fisiológico y psicológico, conocida comúnmente como estrés laboral, la cual conduce a un deterioro progresivo de la salud de las personas durante el ejercicio de su trabajo e incluso fuera de este contexto.

Los efectos sobre la salud del trabajador de los riesgos psicosociales, puede provocar conductas negativas para la organización de trabajo, como el ausentismo, reducción de la satisfacción laboral, mayor índice de accidentabilidad y propensión a abandonar la institución, como consecuencia del malestar psicológico, esto también puede afectar, la atención que brinda el personal de salud a la población usuaria. Teniendo en cuenta lo referido, la investigación en el sector del personal trabajador de salud se vuelve una tarea de primera orden, siendo importante, resaltar que todo el público en general puede llegar a presentar estos síntomas y signos de malestar psicológico; ante ello, se plantea la relevancia de abordar estos temas, para conocer y ahondar en la prevención de los efectos negativos de los trastornos psicológicos en el ámbito laboral, en conjunto con estas afecciones, se hace una extensión sobre el estudio de las consecuencias negativas del consumo de alcohol, cuestión que no podía dejarse de lado en este dominio a investigar como sintomatología de salud mental en trabajadores de un hospital público.

Según el Reporte Mundial de la Salud (2013), los trastornos mentales han ido 
incrementándose en los últimos años, considerándose como las enfermedades que reportan mayor carga en términos de años de vida saludables perdidos, por discapacidad o muerte prematura. Por ejemplo, la depresión, es considerada como la más importante discapacidad, que normalmente se asocia con ansiedad, siendo prevalente entre las edades de 15 y 44 años, edades de población potencialmente trabajadora. La prevalencia de estas afecciones se ha evidenciado mayormente en mujeres y mayores de edad; de manera similar se presenta en el consumo de psicotrópicos. En Latinoamérica según Mingote (2011), las licencias médicas por "trastornos mentales y de comportamiento" incrementaron un alza del $82 \%$ entre 2005 y 2007; siendo las patologías asociadas la depresión, ansiedad y estrés, en el tramo de 20 y 40 años, esta situación es frecuente en profesionales de la salud, laborando bajo presión, en confinamiento, manteniendo un contacto directo con el usuario y sus dolencias, circunstancias que ponen en riesgo de sufrir alguno de estos trastornos emocionales (Abreu, 2011; Vargas y Días, 2011). También se debe indicar el trabajo de enfermería, que se le ha considerado como una profesión con alta prevalencia de trastornos psicológicos, que repercuten negativamente en su salud y calidad de vida, provocando ausentismo laboral y afectación en su calidad de trabajo (Lerma, Rosales y Gallegos, 2009).

El interés planteado en el estado de profesionales de salud, ha crecido considerablemente, los últimos años, las investigaciones se han centrado en los servicios de emergencia y de vigilancia intensiva (Segura et al, 2006), estos estudios han presentado los síntomas de los trastornos de ánimo, en concreto de la ansiedad y la depresión, como consecuencias del síndrome de Burnout (Ortega y López, 2004), aunque esta relación no está del todo clara; en el presente trabajo, se estudian los síntomas de ansiedad y la depresión como síntomas independientes del estrés. Son conocidas las repercusiones negativas que tienen la ansiedad y la depresión en la calidad de vida y en el desempeño socio-ocupacional (Rojtenberg, 2001). Por último, el Consejo de Administración de la OIT (Organización Internacional del Trabajo) aprobó el 25 de marzo del 2010 la lista de enfermedades profesionales, en la que se incluían por primera vez los trastornos mentales y del comportamiento. Al respecto, se ha puesto en alto riesgo psicosocial a los trabajadores de servicios asistenciales y de manera particular a los de servicios sanitarios (Gil, 2009). Como se mencionó anteriormente, este trabajo no deja de lado el consumo de alcohol, dado que, los estudios concluyen que el $20 \%$ de trabajadores consumen bebidas alcohólicas y otras sustancias de manera inapropiada, relacionado con un alto índice de perdida de productividad y afecciones relacionadas al consumo (Mingote, 2011; Enríquez et al, 2011).

\section{Metodología}

La investigación es de tipo cuantitativo, transversal, analítico y de nivel descriptivo (Supo, 2013). También corresponde al tipo no experimental con un diseño transeccional, dado que, recoge los datos en un solo momento; con el propósito de describir sus variables y analizar la incidencia de es estos (Hernandez, Fernandez y Baptista, 2010). Este diseño descriptivo indaga la incidencia de las modalidades, categorías o niveles de una o más variables; en este caso describir la Sintomatología de Salud mental, es decir, ansiedad, depresión, estrés y consumo de alcohol. 


\section{Participantes}

Se analizó la muestra mediante un muestreo aleatorio simple, siendo la muestra un total de 255 trabajadores del Hospital Regional del Cusco, que posteriormente se redujo a 235 por la invalidación de protocolos. Según el sexo, el $77 \%$ son de sexo femenino y el $23 \%$ masculino; según el estado civil el 43,4\% es casado(a), el 32,3\% es soltero(a), el 11,5\% no indicó su estado civil, el 7,2 \% es conviviente, el 4,7\% es divorciado(a) y el $0.9 \%$ es viudo(a). Respecto a los servicios, la distribución se presenta en la siguiente tabla 1 :

\section{Tabla 1}

Servicios del Hospital Público

\begin{tabular}{lcr}
\hline & $\mathrm{N}$ & $\%$ \\
\hline PROCETSS & 4 & 1,7 \\
UCI & 14 & 6,0 \\
Farmacia & 24 & 10,2 \\
Laboratorio & 5 & 2,1 \\
Ginecología & 7 & 3,0 \\
Lavandería & 11 & 4,7 \\
Medicina A & 14 & 6,0 \\
Esclusa & 7 & 3,0 \\
Emergencia & 29 & 12,3 \\
Mantenimiento & 36 & 15,3 \\
Sala de operación & 19 & 8,1 \\
Medicina B & 8 & 3,4 \\
Pediatría B & 12 & 5,1 \\
Cirugía A & 6 & 2,6 \\
Nutrición y dieta & 16 & 6,8 \\
Maternidad & 16 & 6,8 \\
Unidad de quemados & 7 & 3,0 \\
\hline Total & 235 & 100,0 \\
\hline
\end{tabular}

\section{Procedimiento de Recolección de Datos}

La técnica de recogida de los datos fue un cuestionario autoadministrado que se aplicó a una muestra de los trabajadores de la institución. La aplicación se efectuó durante los meses de Setiembre y Octubre del 2016 en el hospital, el cual se ubica en la ciudad del Cusco, Perú. A cada participante se le solicito firmar una hoja de consentimiento siguiendo los protocolos de consideraciones éticas.

\section{Tratamiento y Análisis de Datos}

En el tratamiento estadístico de la información se utilizó el paquete Statistical Package for the Social Siences (SPSS - 23) las pruebas estadísticas utilizadas en el estudio fueron: estadísticas de resumen y frecuencias. Dado que las variables depresión, ansiedad y estrés no presentan distribución normal se optó por el uso de pruebas no paramétricas. 
Instrumentos o Medidas

Los participantes respondieron a los siguientes cuestionarios:

\section{Para la Ansiedad: Inventario de Ansiedad Estado-Rasgo (STAI) (Spielberger, Gorsuch y Lushene, 1979).}

Consta de 40 ítems que comprende dos subescalas de 20 ítems, una mide la ansiedad estado (A-E) y otra la ansiedad rasgo (A-R). Comprende una escala tipo Likert de cuatro opciones de respuestas que cambian en cada subescala (AE: nada, algo, bastante, mucho; A-R: casi nunca, a veces, a menudo, casi siempre). El rango de las puntuaciones varía entre 0 y 60 en cada escala. El coeficiente alfa de los estudios originales oscila entre 0.83 y 0.92 (Spielberger et al., 1970).

Para Depresión: Beck Depression Inventory (BDI) de Beck, Ward, Mendelson, Mock y Erbaugm (1961) adaptado por Sanz y Vázquez (1998).

Considerado uno de los instrumentos más utilizados para evaluar los síntomas de depresión, mediante 21 ítems con opciones de respuestas de 0 a 3 referidas a la intensidad de síntomas cognitivos, fisiológicos y conductuales del estado de ánimo deprimido, obteniendo puntuaciones de 0 a 63 con diferentes valores de corte (Beck, Steer y Garbin, 1988).Así, una puntuación total de 0 a 9 indicaría ausencia de depresión, de 10 a 18 indicaría depresión leve, de 19 a 29 depresión moderada y por último, una puntuación mayor de 30 indicaría depresión grave. Las propiedades psicométricas del cuestionario han demostrado adecuados índices de fiabilidad en la versión original (Beck et al., 1988) como en la versión española. (Sanz y Vázquez, 1998).

Para el estrés: La escala «the stress scale» (escala de estresores laborales) fue diseñada por Gray-Toft et al. para valorar los estresores laborales (factores causales de estrés) en personal de instituciones de servicios.

El instrumento comprende 48 estresores (ítems) elaborados a partir de los estudios y entrevistas hechas a trabajadores y empleados; donde $0=$ nunca, $1=$ alguna vez, 2 = frecuentemente, 3 = muy frecuentemente, así, la puntuación va desde 0 como ausencia de estrés a 144 indicando alto nivel de estrés. Esta escala se diseño para ser autoadministrada, requiriendo un tiempo de $10 \mathrm{~min}$ para su llenado. La escala muestra una fiabilidad (reproducibilidad) un coeficiente alfa de Cronbach superior a 0,7. Ello sugiere que los ítems que integran el total de la escala o cada una de las subescalas están midiendo un constructo unitario.

Para alcoholismo: Alcohol Use Disorders Identification Test (AUDIT). Creado por Saunders, Aasland, Babor, De La Fuente y Grant, 1992. Adaptado al español en 1998.

Está basado en un proyecto de la Organización Mundial de la Salud (OMS), que fue posteriormente estandarizado por Saunders et al 1992. El AUDIT sigue los criterios del DSM-IV la CIE-10, resultando efectivo siguiendo también los criterios de la CIE-10 sobre problemas relacionados con el consumo alcohólico. Los ítems evalúan la cantidad y frecuencia del consumo de alcohol, dificultad de controlar la bebida, abandono de aficiones y síntomas de abstinencia, reacciones adversas como problemas en el funcionamiento derivados del consumo de alcohol y problemas relacionados con el consumo de alcohol. Posee una Consistencia 
interna: 0,80. Siguiendo los criterios de la OMS sobre problemas relacionados con el consumo de alcohol, si se establece un punto de corte de 11 o más, la sensibilidad es de 0,84 y la especificidad de 0,71. Un punto de corte de 13 o más proporciona una sensibilidad de 0,7 y una especificidad del 0,78 .

\section{Resultados}

\section{Nivel de ansiedad, depresión, estrés y alcoholismo}

Los niveles en ansiedad se representan por dos valores, el primero sobre Ansiedad de Estado arrojando en un 94,9\% con nivel alto, segundo, el 44,7 \% está en el nivel sobre promedio de Ansiedad de Rasgo, concluyendo que el nivel de Ansiedad es elevado en los trabajadores del Hospital. Respecto al nivel de depresión se evidencia en un $62,6 \%$ la no presencia de sintomatología depresiva, sin embargo, el $31,5 \%$ se encuentra con depresión leve y el $6 \%$ con depresión moderada, no llegando a síntomas depresivos graves. En los niveles de estresores laborales los resultados arrojan evidencia de que el $37,9 \%$ presenta un nivel de estrés manejable con sus propios recursos. Y, por último, el 93.6\% no presenta problemas relacionados con la bebida, no obstante, el $4.7 \%$ tiene condición de bebedor de riesgo, y el $4 \%$ puede presentar dependencia alcohólica. 
Tabla 2

Niveles de ansiedad, depresión, estrés y alcoholismo

\begin{tabular}{|c|c|c|}
\hline \multicolumn{3}{|l|}{ Nivel Ansiedad Estado } \\
\hline & Frecuencia & Porcentaje \\
\hline Alto & 223 & 94,9 \\
\hline Sobre Promedio & 8 & 3,4 \\
\hline Tendencia a Promedio & 4 & 1,7 \\
\hline Total & 235 & 100,0 \\
\hline \multicolumn{3}{|l|}{ Nivel Ansiedad Rasgo } \\
\hline & Frecuencia & Porcentaje \\
\hline Alto & 22 & 9,4 \\
\hline Sobre Promedio & 105 & 44,7 \\
\hline Promedio & 12 & 5,1 \\
\hline Tendencia a Promedio & 49 & 20,9 \\
\hline Bajo & 47 & 20,0 \\
\hline Total & 235 & 100,0 \\
\hline \multicolumn{3}{|l|}{ Nivel Depresión } \\
\hline & Frecuencia & Porcentaje \\
\hline No Depresión & 147 & 62,6 \\
\hline Depresión Leve & 74 & 31,5 \\
\hline Depresión Moderada & 14 & 6,0 \\
\hline Total & 235 & 100,0 \\
\hline \multicolumn{3}{|l|}{ Nivel de Estrés } \\
\hline & Frecuencia & Porcentaje \\
\hline Ausencia de estrés & 10 & 4,3 \\
\hline Nivel minimo de estrés & 66 & 28,1 \\
\hline Estrés manejable & 89 & 37,9 \\
\hline Elevado nivel de estrés & 42 & 17,9 \\
\hline Alto nivel de estrés & 18 & 7,7 \\
\hline Muy alto nivel de estrés & 10 & 4,3 \\
\hline Total & 235 & 100,0 \\
\hline \multicolumn{3}{|l|}{ Alcoholismo } \\
\hline & Frecuencia & Porcentaje \\
\hline No problemas relacionados con la bebida & 220 & 93,6 \\
\hline Bebedor(a) de riesgo & 11 & 4,7 \\
\hline $\begin{array}{l}\text { Problemas fisicos-psiquicos relacionados con la bebida y /o } \\
\text { probable dependencia alcohólica }\end{array}$ & 4 & 1,7 \\
\hline Total & 235 & 100,0 \\
\hline
\end{tabular}

En un análisis exhaustivo de los niveles de ansiedad, depresión, estrés y alcoholismo (gráficos 1, 2, 3, 4 y 5) es posible apreciar que, en su mayoría, tanto en varones $(94,5 \%)$ como en mujeres (96,3\%) presenta Ansiedad de Estado alto. Respecto a Ansiedad de Rasgo muestran tanto en varones (42.6\%) como en mujeres $(45,3 \%)$ los picos más altos en sobre promedio. En depresión se evidencia en un $62,6 \%$ la no presencia de sintomatología depresiva, la diferencia entre varones $(46,3 \%)$ y mujeres $(27,1 \%)$ es evidente en depresión leve. Respecto a los niveles 
de Estrés muestran entre varones (40,7\%) y mujeres (37\%) sus resultados en estrés manejable. En los niveles de alcoholismo se evidencia la diferencia entre varones $(16,7 \%)$ y mujeres $(1,1 \%)$ en la categoría de bebedor(a) de riesgo. Por último, con el objetivo de hacer las precisiones respecto a cada servicio se presenta la evidencia del porcentaje de incidencia de sintomatología en cada servicio.

\section{Figura 1}

Nivel de Ansiedad de Estado respecto al Sexo

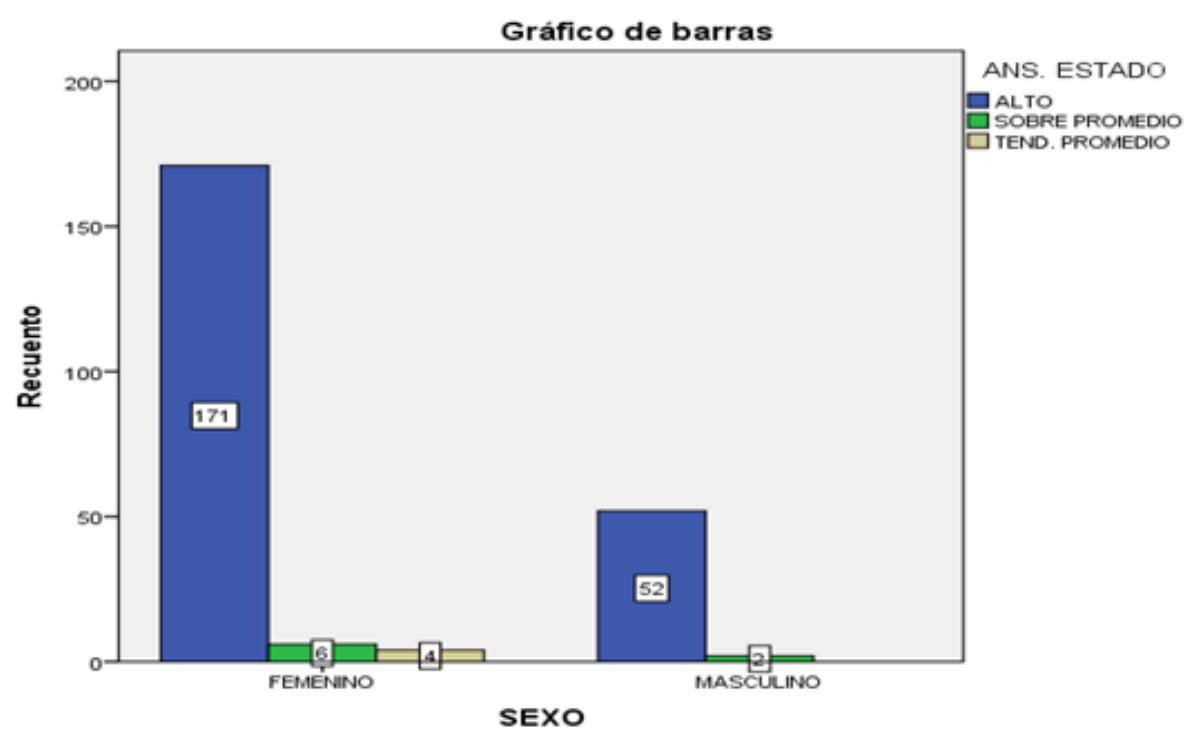

En la figura 1 se puede evidenciar que para en cuestión de sexo, los niveles de ansiedad estado sin altos, tanto para varones como para mujeres, indicando que la presencia de ansiedad es emocionalmente transitorio y fluctuante. 


\section{Figura 2}

Nivel de Ansiedad de Rasgo respecto al Sexo

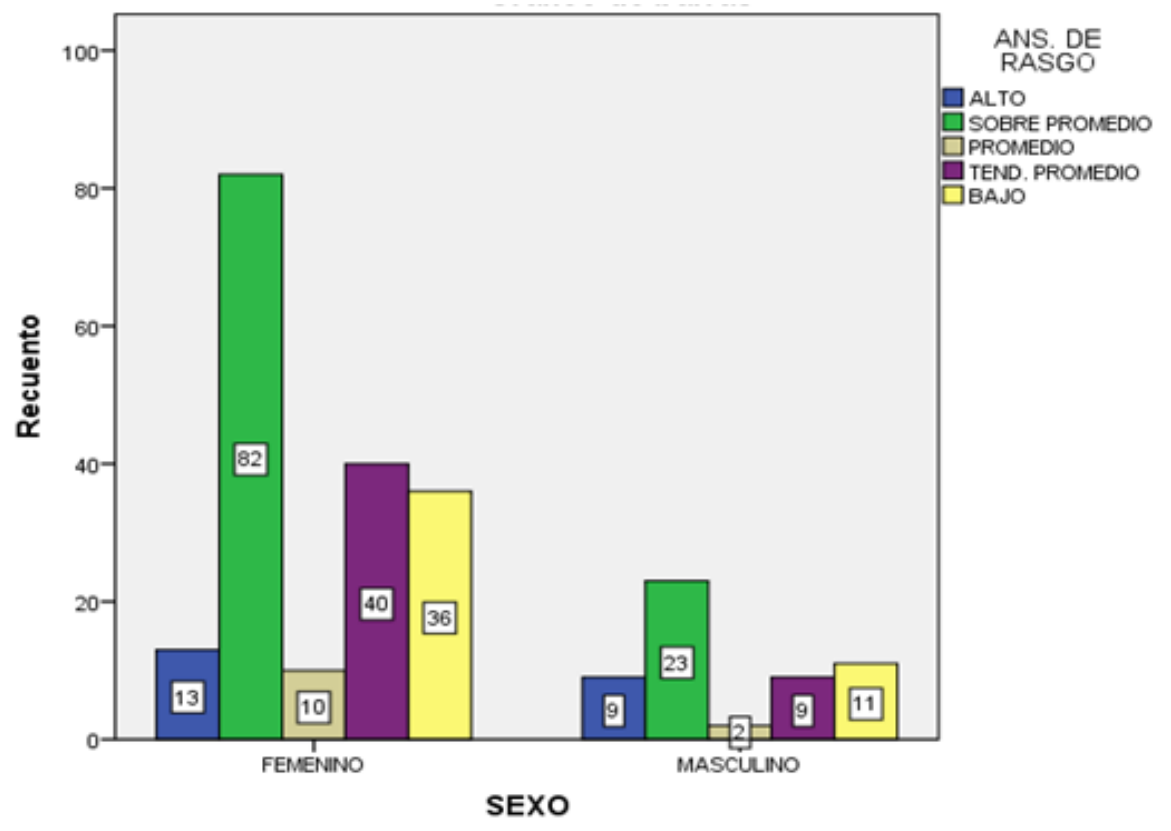

En la figura 2 se observa las frecuencias descriptivas para la ansiedad de rasgo, observándose niveles sobre promedio mayormente reportados, tanto en mujeres como en varones, no obstante, se puede observar en menor cantidad tendencias promedio de ansiedad rasgo, estos resultados indican una predisposición individual a responder de manera ansiosa.

\section{Figura 3}

Nivel de Depresión por Sexo

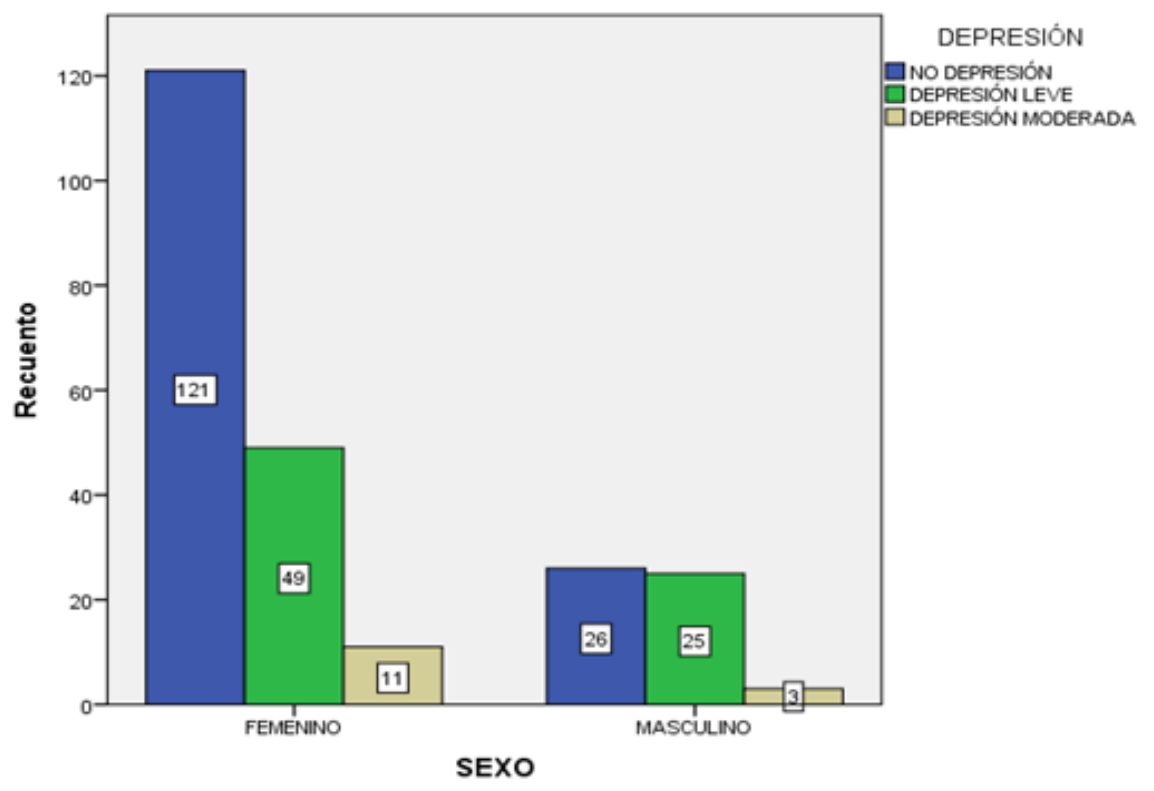


En las frecuencias descriptivas de la variable depresión, observadas en la figura 3 , se evidencia, la no presencia de sintomatología depresiva, no obstante, en menor porcentaje y significativo los niveles de depresión moderada y depresión leve se hacen presentes, tanto en varones como en mujeres, siendo mayor en mujeres.

\section{Figura 4}

Nivel de Estrés por Sexo

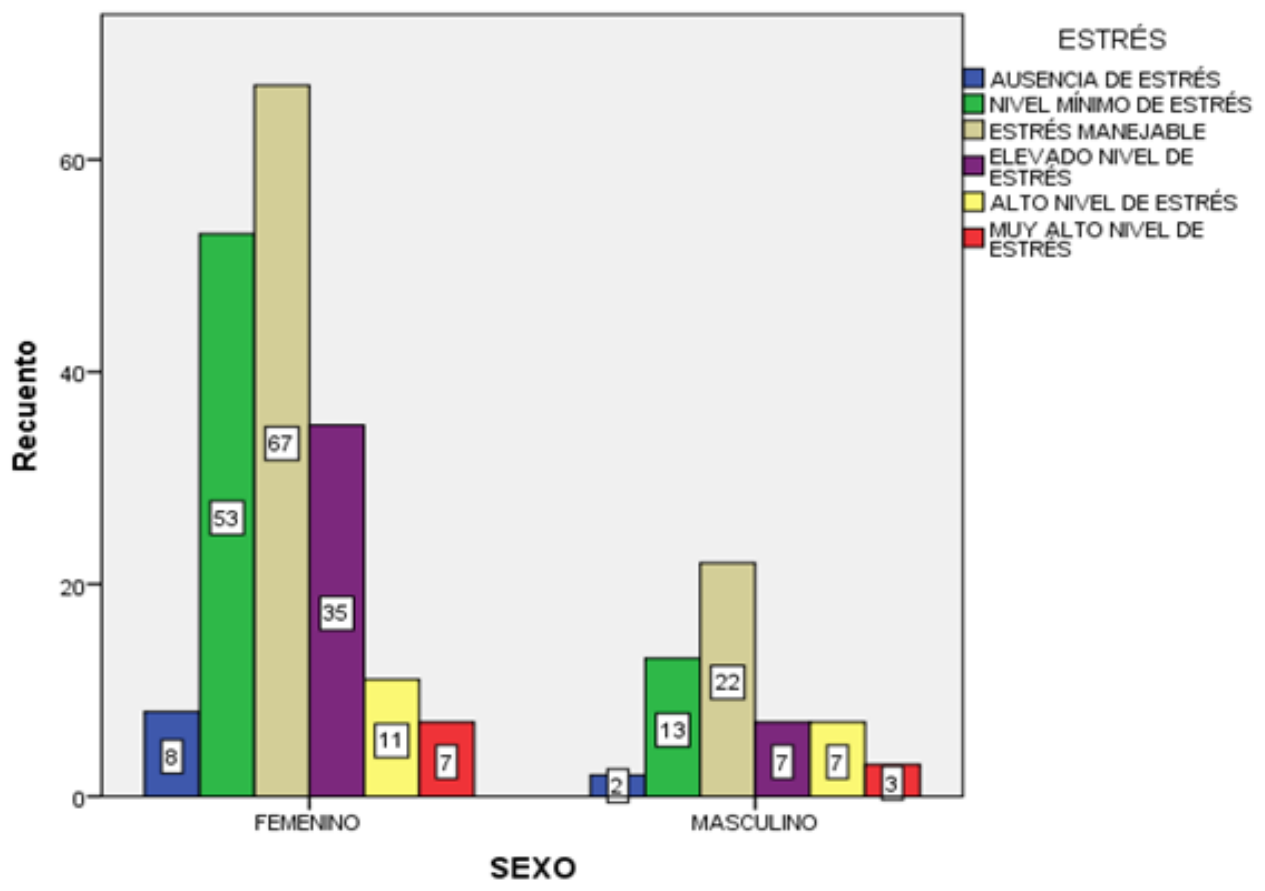

En la figura 4 sobre los niveles de estrés se puede observar que, el reporte mayor se encuentra con niveles manejables de estrés, seguido por niveles bajos de estrés, no obstante, un porcentaje significativo reporta elevado nivel de estrés y alto nivel de estrés, siendo importante focalizar esfuerzos de atención a este personal de salud, a pesar de ello, los índices muestran un estrés manejable mayoritariamente. 


\section{Figura 5}

Nivel de Alcoholismo por Sexo

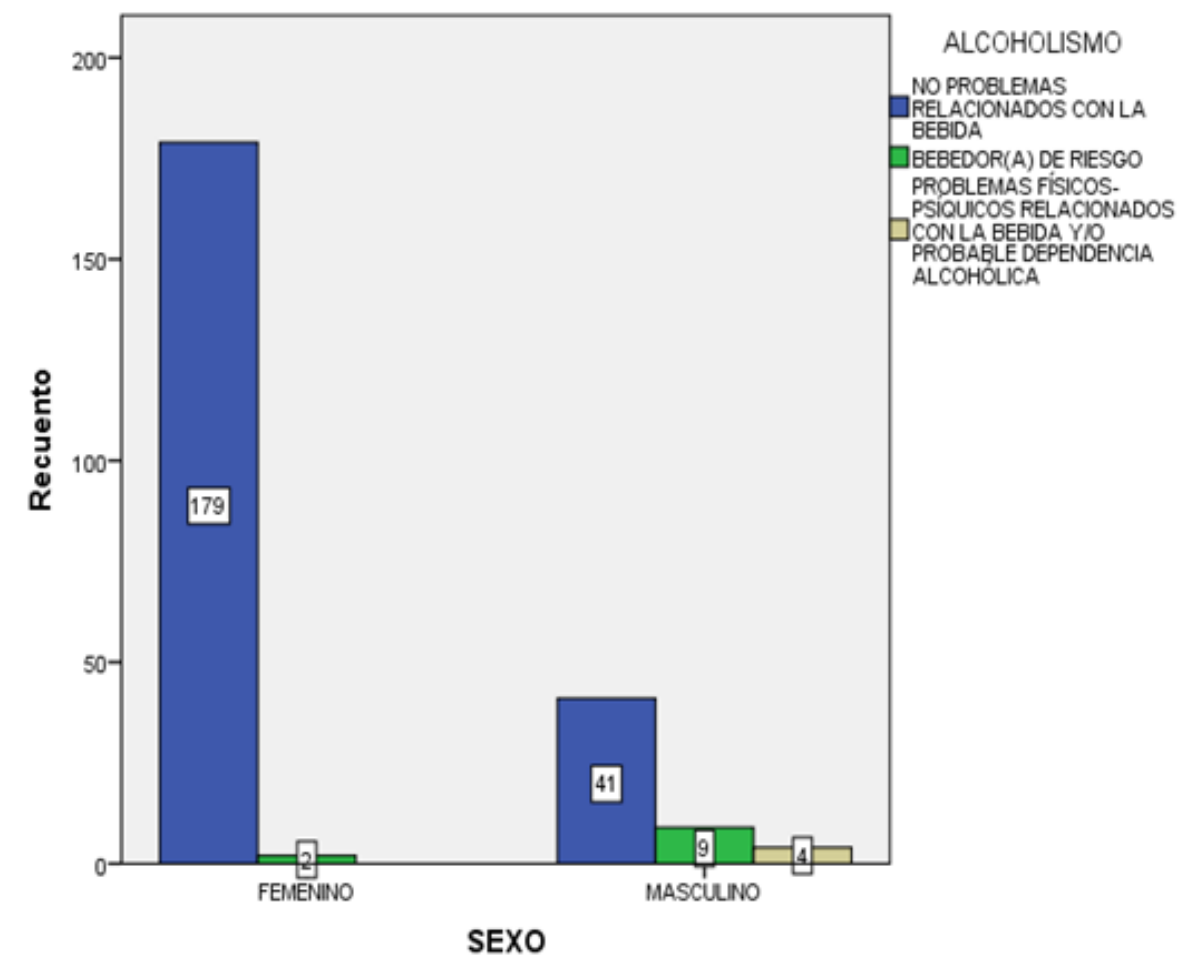

Respecto a la figura 5 sobre los niveles ofrecidos por el AUDIT sobre el consumo de alcohol excesivo, se observa con mayor reporte problemas no relacionados con la bebida, es importante mencionar que hay un reporte muy bajo de bebedores de riesgo, sobre todo en varones, donde habría que poner atención.

\section{Diferencias en los niveles de ansiedad, depresion, estrés y alcoholismo en los servicios}

En la tabla 3 observamos que en un $94.9 \%$ los servicios de salud presentan ansiedad estado, siendo los servicios de PROCETTS, UCI, laboratorio, ginecología, emergencia, sala de operaciones, pediatría, maternidad y unidad de quemados servicios con el $100 \%$ de ansiedad estado y un servicio suplementario de lavandería, demostrando que esta característica es importante para el desempeño de sus labores, no obstante, todos los servicios cuentan con un nivel alto, demostrando la misma capacidad. 
Tabla 3

Porcentajes de Incidencia de Ansiedad Estado en Servicios

\begin{tabular}{lccc}
\hline & Alto & Sobre Promedio & Tendencia Promedio \\
\cline { 2 - 4 } PROCETSS & $100,0 \%$ & & \\
UCI & $100,0 \%$ & $4,2 \%$ & \\
Farmacia & $95,8 \%$ & & \\
Laboratorio & $100,0 \%$ & & \\
Ginecologial & $100,0 \%$ & & \\
Lavanderia & $100,0 \%$ & $14,3 \%$ & \\
Medicina A & $85,7 \%$ & $14,3 \%$ & \\
Escluga & $57,1 \%$ & & $12,5 \%$ \\
Emergencia & $100,0 \%$ & $2,8 \%$ & \\
Mantenimiento y Limpieza & $97,2 \%$ & $12,5 \%$ & \\
Sala de operaciones & $100,0 \%$ & & \\
Medicina B & $75,0 \%$ & $16,7 \%$ & $6,3 \%$ \\
Pediatria B & $100,0 \%$ & $6,3 \%$ & \\
Cirugia A & $83,3 \%$ & & \\
Nutrición y dieta & $87,5 \%$ & & \\
Maternidad & $100,0 \%$ & $3,4 \%$ & \\
Unidad de quemados & $100,0 \%$ & & \\
\hline & $94,9 \%$ & & \\
\hline
\end{tabular}

Por otro lado, la tabla 4 muestra a los servicios con mayor variabilidad de ansiedad rasgo, ubicándose en sobre promedio la mayor cantidad de reporte, superando el $30 \%$ de incidencia hasta un $50 \%$, esto demuestra niveles emocionales fluctuantes considerables de ansiedad en el personal trabajador, siendo significativos en los servicios de PROCETS, UCI, laboratorio, Medicina A y Emergencia, y en lavandería de manera suplementaria. 


\section{Tabla 4}

Porcentajes de Incidencia de Ansiedad Rasgo en Servicios

\begin{tabular}{|c|c|c|c|c|c|}
\hline & & & Ansiedad R & & \\
\hline & Alto & $\begin{array}{c}\text { Sobre } \\
\text { promedio }\end{array}$ & Promedio & $\begin{array}{l}\text { Tendencia } \\
\text { Promedio }\end{array}$ & Bajo \\
\hline PROCETSS & $25,0 \%$ & $50,0 \%$ & $25,0 \%$ & & \\
\hline $\mathrm{UCI}$ & $7,1 \%$ & $50,0 \%$ & $7,1 \%$ & $28,6 \%$ & $7,1 \%$ \\
\hline Farmacia & $16,7 \%$ & $58,3 \%$ & & $12,5 \%$ & $12,5 \%$ \\
\hline Laboratorio & & $60,0 \%$ & & $20,0 \%$ & $20,0 \%$ \\
\hline Ginecologia & $28,6 \%$ & $57,1 \%$ & & $14,3 \%$ & \\
\hline Lavandería & $9,1 \%$ & $54,5 \%$ & & $27,3 \%$ & $9,1 \%$ \\
\hline Medicina A & & $64,3 \%$ & & $21,4 \%$ & $14,3 \%$ \\
\hline Esclusa & $14,3 \%$ & $42,9 \%$ & & $14,3 \%$ & $28,6 \%$ \\
\hline Emergencia & $6,9 \%$ & $51,7 \%$ & $6,9 \%$ & $27,6 \%$ & $6,9 \%$ \\
\hline $\begin{array}{l}\text { Mantenimiento y } \\
\text { Limpieza }\end{array}$ & $11,1 \%$ & $30,6 \%$ & $8,3 \%$ & $167 \%$ & $33,3 \%$ \\
\hline Sala de operación & $5,3 \%$ & $31,6 \%$ & & $36,8 \%$ & $26,3 \%$ \\
\hline Medicina B & $25,0 \%$ & $37,5 \%$ & & & $37,5 \%$ \\
\hline Pediatria B & & $58,3 \%$ & & $16,7 \%$ & $25,0 \%$ \\
\hline Cirugia A & & $33,3 \%$ & $33,3 \%$ & $33,3 \%$ & \\
\hline Nutrición y dieta & & $31,3 \%$ & & $25,0 \%$ & $43,8 \%$ \\
\hline Maternidad & $12,5 \%$ & $31,3 \%$ & $12,5 \%$ & $25,0 \%$ & $18,8 \%$ \\
\hline Unidad de quemados & $14,3 \%$ & $42,9 \%$ & $14,3 \%$ & & $28,6 \%$ \\
\hline Total & $9,4 \%$ & $44,7 \%$ & $5,1 \%$ & $20,9 \%$ & $20,0 \%$ \\
\hline
\end{tabular}

En la tabla 5, observamos que la depresión está presente con niveles leves y moderados en los servicios de PROCETSS de atención a pacientes con VIH y enfermedades de transmisión sexual, en el servicio de Farmacia, en medicina A, Emergencia con menor reporte, en Nutrición de manera considerable, en el personal de mantenimiento con reporte considerable y maternidad. 


\section{Tabla 5}

Porcentaje de Incidencia de Depresión en Servicios

\begin{tabular}{lccc}
\hline & \multicolumn{3}{c}{ Depresión } \\
\cline { 2 - 4 } & No Depresión & Depresión Leve & Depresión Moderada \\
\cline { 2 - 4 } PROCETSS & $21,4 \%$ & $100,0 \%$ & \\
UCI & $4,2 \%$ & $78,6 \%$ & $4,2 \%$ \\
Farmacia & $100,0 \%$ & $91,7 \%$ & \\
Laboratorio & $85,7 \%$ & & \\
Ginecologia & $63,6 \%$ & $14,3 \%$ & $14,3 \%$ \\
Lavanderia & $71,4 \%$ & $36,4 \%$ & \\
Medicina A & $100,0 \%$ & $14,3 \%$ & $3,4 \%$ \\
Esclusa & $69,0 \%$ & $27,6 \%$ & $11,1 \%$ \\
Emergencia & $63,9 \%$ & $25,0 \%$ & \\
Mantenimiento y & $78,9 \%$ & $21,1 \%$ & $12,5 \%$ \\
Limpieza & $75,0 \%$ & $12,5 \%$ & \\
Sala de operación & $91,7 \%$ & $8,3 \%$ & \\
Medicina B & $100,0 \%$ & & $25,0 \%$ \\
Pediatría B & $50,0 \%$ & $25,0 \%$ & $6,3 \%$ \\
Cirugia A & $75,0 \%$ & $18,8 \%$ & \\
Nutrición y dieta & $100,0 \%$ & & $6,0 \%$ \\
Maternidad & $62,6 \%$ & $31,5 \%$ & \\
Unidad de quemados & & & \\
\hline Total & & & \\
\hline
\end{tabular}

La tabla 6 muestra las frecuencias de presencia de estrés, es importante resaltar los porcentajes ubicados en elevado, alto y muy alto, dado que, indican presencia de síntomas del síndrome de Burnout, donde encontramos al servicio de UCI, Farmacia, Lavandería, Medicina, esclusa, emergencia, Mantenimiento y limpieza, Pediatría, nutrición y dieta y maternidad. Sin embargo, como ya mencionamos, los niveles de mayor reporte se encuentran en estrés manejable. 
Tabla 6

Porcentajes de Incidencia de Estrés en Servicios

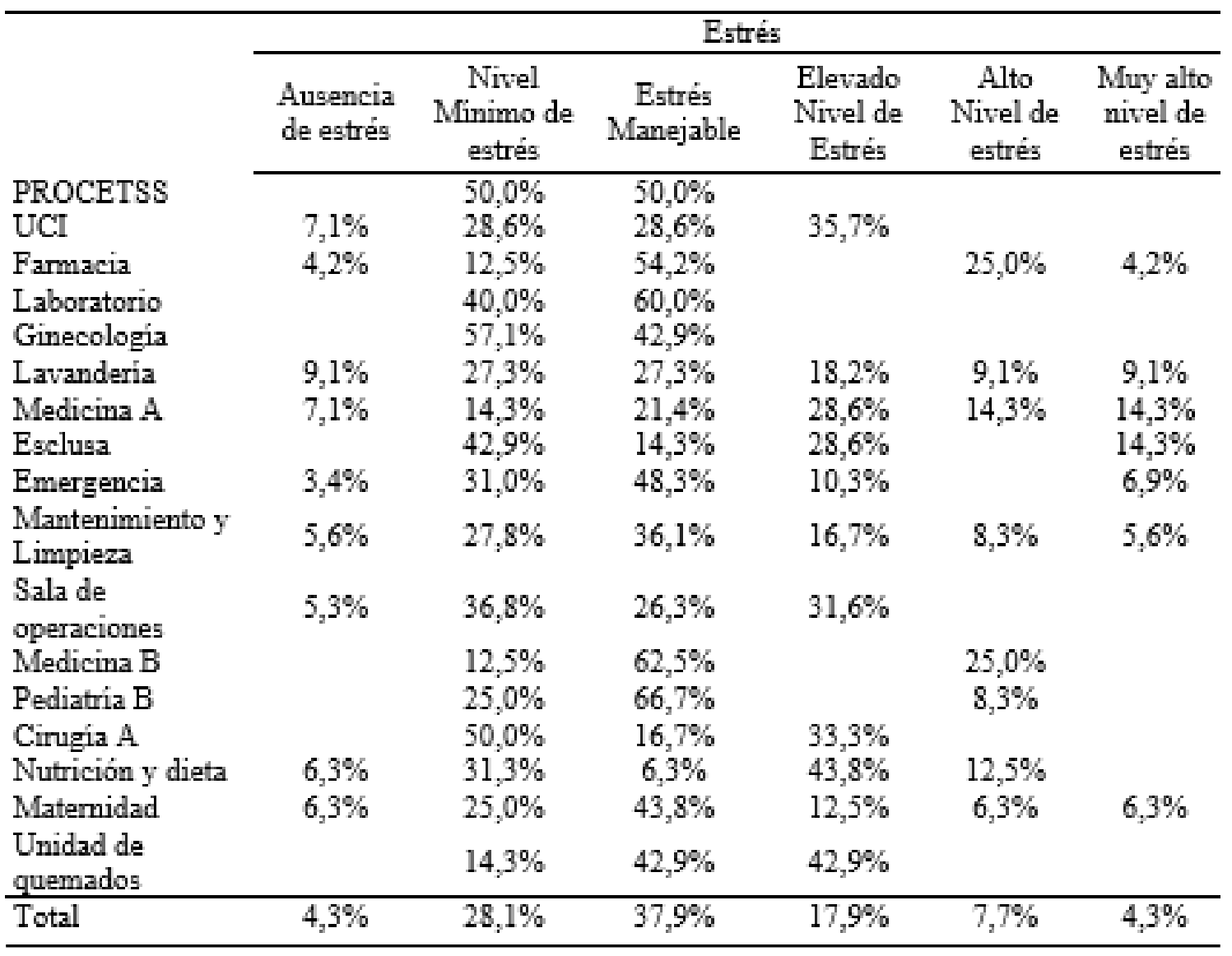


Tabla 7

Porcentaje de alcoholismo por servicios

\begin{tabular}{|c|c|c|c|}
\hline & \multicolumn{3}{|c|}{ Alcoholismo } \\
\hline & $\begin{array}{l}\text { No problemas } \\
\text { relacionados con la } \\
\text { bebida }\end{array}$ & $\begin{array}{c}\text { Bebedor (a) de } \\
\text { Riesgo }\end{array}$ & $\begin{array}{l}\text { Problemas físicos- } \\
\text { paíquicos } \\
\text { relacionados con la } \\
\text { bebida y/o probable } \\
\text { dependencia } \\
\text { alcohólica }\end{array}$ \\
\hline PROCETSS & $100,0 \%$ & & \\
\hline $\mathrm{UCI}$ & $100,0 \%$ & & \\
\hline Farmacia & $91,7 \%$ & & $8,3 \%$ \\
\hline Laboratorio & $100,0 \%$ & & \\
\hline Ginecologia & $100,0 \%$ & & \\
\hline Lavanderia & $72,7 \%$ & $27,3 \%$ & \\
\hline Medicina A & $100,0 \%$ & & \\
\hline Esclusa & $100,0 \%$ & & \\
\hline Emergencia & $93,1 \%$ & $6,9 \%$ & \\
\hline Mantenimiento y Limpieza & $80,6 \%$ & $13,9 \%$ & $5,6 \%$ \\
\hline $\begin{array}{l}\text { Sala de operación } \\
\text { Medicina B }\end{array}$ & $\begin{array}{l}100,0 \% \\
100,0 \%\end{array}$ & & \\
\hline Pediatria B & $100,0 \%$ & & \\
\hline Cirugia A & $100,0 \%$ & & \\
\hline Nutrición y dieta & $100,0 \%$ & & \\
\hline Matemidad & $93,8 \%$ & $6,3 \%$ & \\
\hline Unidad de quemados & $100,0 \%$ & & \\
\hline Total & $93,6 \%$ & $4,7 \%$ & $1,7 \%$ \\
\hline
\end{tabular}

\section{Discusión}

En esta investigación se identificaron los niveles de depresión, ansiedad y estrés en los trabajadores de la salud; adicionalmente, se analizó la existencia de diferencias estadísticamente significativas entre el género y dichas sintomatologías de salud mental. El resultado principal de este trabajo es que la mayoría $(>70 \%)$ de los participantes de este estudio, tanto hombres como mujeres, poseen ansiedad tanto de estado como de rasgo entre alto y sobre promedio. Este resultado coincide con la investigación realizada por Ayuso, Colomer, Herrera, 2016, en cuyo reporte de la ansiedad también se manifiestan en rango similares; en contraste con lo que ocurre en trabajos con personal de enfermería en donde fueron encontrados valores más altos en ansiedad de rasgo (Hernández, 2003), lo cual podría ser aceptable dado que el personal sanitario debe mantenerse activo ante situaciones de riesgo ante la responsabilidad de atender adecuadamente a los pacientes. Quedaría para posteriores investigaciones si este nivel de ansiedad es signo de un desgaste emocional, sin embargo, y en relación a la depresión los trabajadores no mostraron en más del $85 \%$ sintomatología depresiva en contraste a la investigación anteriormente referida por 
García y Castañeda, 2011. Sobre los estresores laborales había mencionado sus implicancias perjudiciales en el entorno de trabajo, las relaciones interpersonales y el sentimiento de vulnerabilidad (Hernández, 2003), sin embargo los resultados mostraron su evidencia en un $37.9 \%$ en estrés manejable y el resto dentro de un nivel de estrés mínimo, lo cual es positivo para dicha institución y dentro de estos elementos positivos están los resultados de alcoholismo en un 93,6 \% son no consumidores de Bebidas alcohólicas rescatando los lineamientos de prevención dados en Ochoa \& Madoz, 2008.

Referente a las diferencias entre el género y los niveles de depresión, ansiedad y estrés en los profesionales de sanitarios estudiados se encontraron diferencias estadísticas que no son significativas al comparar las medias de los grupos (femenino y masculino); situación que contrasta con el estudio de Hernández (2003) en el cual se encontró que los síntomas de estrés con valores promedio significativamente mayores en las mujeres y significantemente mayores en la categoría de bebedores de riesgo en varones. Los resultados aquí expuestos deberán tomarse con reserva ya que en la investigación la selección de los trabajadores se realizó a través de un muestreo por conveniencia y no se tiene la seguridad de que cada unidad maestral integre a la población total en el proceso de selección de la muestra. Además, estos hallazgos deben interpretarse como una tendencia de la situación, por tanto, no informan las razones de los padecimientos; de ahí que, estas particularidades constituyen limitantes a este estudio. No obstante, la información obtenida es un buen marco de referencia para investigaciones posteriores.

Por último, los resultados, responden a los objetivos planteados, dado que conocemos la incidencia de sintomatología de salud mental en el personal sanitario en ansiedad, depresión, estrés y alcoholismo, siendo la conclusión que el personal tiene ansiedad de estado y rasgo elevados, no presenta sintomatología de depresiva, sus niveles de estrés son manejables con recursos propios del personal sanitario y no hay consumo perjudicial en más del $80 \%$ del personal sanitario y trabajador, aunque hay que recordar que el personal trabajador de la unidad de Mantenimiento, Conservación, limpieza y Seguridad tienen un índice del 19,4 \% entre bebedores de riesgo y consumo perjudicial, otra unidad con alto nivel en esta categoría es el servicio de lavandería con $27,3 \%$, que para que se cumpla con los lineamientos de un buen ámbito laboral planteado por la OIT se recomienda que deberían ser abordados. Es importante recalcar que respondiendo los objetivos de la investigación se evidencia grandes diferencias entre cada uno de los servicios respecto al nivel de sintomatología de salud mental, en fin, se concluye que se ha respondido a cada uno de los objetivos planteado por el investigador, recabando información importante para la salud mental en personal sanitario y trabajador de un hospital público de la ciudad del Cusco.

Sin embargo, a pesar de la búsqueda bibliográfica realizada, se han encontrado pocos estudios que traten sobre la prevalencia de síntomas de depresión y la ansiedad en nuestro país y ninguno en profesionales trabajadores generales de hospitales. En uno de estos estudios, por ejemplo, el de Caballero et al, 2001 en el que participaron (médicos, enfermeras/os y auxiliares) de un área hospitalaria de Madrid, se encontró que entre los síntomas o patologías que reconocían los encuestados destacaban los referidos a la ansiedad y depresión. En otro trabajo realizado en las Unidades Coronaria y 
Cardiovascular del Hospital Clínico Universitario de Salamanca llevado a cabo por García et al, 2008 en donde se evaluó la ansiedad (STAI), los resultados mostraron que el $37.5 \%$ de los profesionales presentaba niveles altos de ansiedad-rasgo y el $45.8 \%$ de ansiedad-estado.

También se han encontrado trabajos de estas características en otros países. En Latinoamérica, se han realizado diversos estudios en los que participaban personal de enfermería y residentes de distintas especialidades. En estos, se ha observado que entre un $30.2 \%$ (STAI) y un $43.3 \%$ (HARS) de los profesionales presentaban síntomas de ansiedad y entre un 13 (BDI) y un 44.4\% (HDRS), de depresión (Betancourt, Rodríguez, Vallecillo, Sánchez, 2008; Lerma, Rosales, Gallegos, 2009; Pérez, 2006; Rosales, Gallardo, Conde, 2005). En EE.UU., el trabajo de Collier, McCue, Markus y Smith (2002) mostró una prevalencia de síntomas de depresión del 35\% en una muestra de 4128 médicos residentes de distintas especialidades. Por otra parte, un estudio realizado en Turquía en este caso con médicos del servicio de urgencias, arrojaron síntomas de ansiedad y depresión (BDI y BAI) en un $14.6 \%$ y $15.1 \%$ respectivamente (Erdur et al., 2006). En otro trabajo, también realizado en el servicio de urgencias, pero en este caso con personal de enfermería, los resultados muestran que un $54 \%$ de las personas referían ansiedad moderada o grave (Faces Anxiety Scale) (Cooke, Holzhauser, Jones, Davis, Finucane, 2007).

\section{Declaración de conflicto de interés}

El autor declara no tener ningún tipo de conflicto de intereses 


\section{Referencias}

García-Rivera, Blanca, Maldonado-Radillo, Sonia, \& Barón, María Ramírez. (2014). Estados afectivos emocionales (depresión, ansiedad y estrés) en personal de enfermería del sector salud pública de México. Summa psicológica UST (En línea), 11(1), 65-72. Recuperado en 06 de Enero de 2017, de http://pepsic.bvsalud. org/scielo.php?script=sci_arttext\&pid=S0719-448x2014000100006\&lng= pt\&tlng=es.

Abizanda-Campos, R; Gómez-Martínez, S; Ballester-Arnal, R; Gil-Julia, B; (2015). Ansiedad, depresión y malestar emocional en los profesionales sanitarios de las Unidades de Cuidados Intensivos. Anales de Psicología, 31, 743-750. doi:http:// dx.doi.org/10.6018/analesps.31.2.158501

Beck, A. T., Steer, R. A. \& Carbin, M. G. (1988). Psychometric properties of the Beck Depression Inventory: Twenty-five years of evaluation. Clinical Psychology Review, 8, 77-100.

Mingote Adán, José Carlos, Pino Cuadrado, Pablo del, Sánchez Alaejos, Raquel, Gálvez Herrer, Macarena, \& Gutiérrez García, Ma Dolores. (2011). El trabajador con problemas de salud mental: Pautas generales de detección, intervención y prevención. Medicina y Seguridad del Trabajo, 57(Supl. 1), 188-205. https://dx. doi.org/10.4321/S0465-546X2011000500012

Beck, A. T., Ward, C. H., Mendelson, M., Mock, J. \& Erbaugm, J. (1961). An inventory for measuring depression. Archives of General Psychiatry, 4, 561-571.

Houtman I, Kompier M. Trabajo y Salud Mental. Enciclopedia de Salud y Seguridad en el Trabajo. oit: Madrid; 2001. Gil PR. Algunas razones para considerar los riesgos psicosociales en el trabajo y sus consecuencias en la salud pública. Rev Esp Salud Pública. 2009; 83(2): 169-173.

Mingote JC. Satisfacción, estrés laboral y calidad de vida del médico. Tesis Doctoral. Universidad Autónoma de Madrid; 1995.

Mingote JC, Gálvez M, Del Pino P, Gutiérrez MD. El paciente que padece un trastorno depresivo en el trabajo. Med Seg Tr 2009; 55(214): 41-43.

Phisycian Health Services, Inc. Massachusetts. Disponible en: http:/www.massmed.org/ Content/NavigationMenu6/HelpingYourselforaColleague/Signs_of_Concern_ PH.htm

Muñoz PE.(2005) Epidemiología de los trastornos mentales. En: Vallejo, Leal (Dir). Tratado de Psiquiatría Vol. I. Barcelona: Ars Médica.

Gil-Monte, P. (2010). Situación actual y perspectiva de futuro en el estudio del estrés laboral, Informació psicológica, No. extra, 100, pp. 68-83. Recuperado de: http:// dialnet.unirioja.es/servlet/articulo? codigo $=3642757$

Abreu, F.J. (2011). Síndrome de agotamiento emocional en profesionales de la unidad de 
cuidados intensivos, Revista Multiciencias, 11(4), 370-377. Recuperado de: http:// www.redalyc.org/articulo.oa?id $=90421972006$

Neffa J. (2003) El trabajo humano. Contribuciones al estudio de un valor que permanece. Buenos Aires: Grupo Editorial Lumen; 204-205.

Segura, B. J., Ferrer, V. M., Palma, S. C., Ger, C. S., Domènech, C. M., Gutiérrez, B. I. \& Cebriá, A. J. (2006). Valores personales y profesionales en médicos de familia y su relación con el síndrome de burnout. Anales de Psicología, 22, 45-51.

Castillo-Carniglia, A; Ansoleaga M, E; (2011). Riesgo psicosocial laboral y patología mental en trabajadores de hospital. Revista Facultad Nacional de Salud Pública, 29()372-379. Recuperado de http://www.redalyc.org/articulo.oa?id=12021522003

Pan American Health Organization. Mental Health in Latin American and the Caribbean. paho and the formulation of Mental Health in the Americas [internet]. paho ;2001 [acceso 05 de enero de 2017]. Disponible en: http://www.paho.org/English/DD/ PIN/ mentalhealth_002.htm.

Spielberger, C., Gorsuch, R \& Lushene, R. (1970). Manual for the State-Trait Anxiety Inventory. Palo Alto, California: Consulting Psychologist Press

Caballero, M. A., Bermejo, F., Nieto, R. \& Caballero, F. (2001). Prevalencia y factores asociados al burnout en un área de salud. Atención Primaria, 5, 313-317

Yerkes, R. M. \& Dodson, J. D. (1908). The Relation of Strength of Stimulus to Rapidity of Habit-Formation. Journal of Comparative Neurology and Psychology, 18, 459482.

García, M., Azpiroz J., De la Fuente, Y., García, M. \& González, B. (2007- 2008). La ansiedad en el personal sanitario. Enfermería en Cardiología, 42- 43, 41-45.

Erdur, B., Ergin, A., Turkcuer, I., Parlak, I., Ergin, N. \& Boz, B. (2006). A study of depression and anxiety among doctors working in emergency units in Denizli, Turkey. Emergency Medicine Journal, 23, 759-763.

Ochoa Mangado, Enriqueta, \& Madoz Gúrpide, Agustín. (2008). Consumo de alcohol y otras drogas en el medio laboral. Medicina y Seguridad del Trabajo, 54(213), 25-32. Recuperado en 06 de enero de 2017, de http:/scielo.isciii.es/scielo. php?script $=$ Sci arttext\&pid $=$ S0465-546X2008000400003\&lng=es\&tlng $=e s$

OIT. Iniciativas en el lugar de trabajo para prevenir y reducir los problemas causados por el alcohol y otras drogas. Ginebra 1988.

OIT. Proyecto de repertorio de recomendaciones prácticas sobre el tratamiento en los lugares de trabajo de los problemas que plantea el consumo de alcohol y drogas. Ginebra 1994 
Martínez, P; Rivera, E; Medina, M E; (2004). Consumo de alcohol y drogas en personal de salud: algunos factores relacionados. Salud Mental, 27() 17-27. Recuperado de http://www.redalyc.org/articulo.oa?id=58262703

OIT. (1998) Lucha contra el abuso de alcohol y drogas en el lugar de trabajo. Ginebra

Díaz C, Lourdes, Ulloa, Célia Marina, Taubert De Freitas, Fabiana, Amorim A, Liliana, Barcelos D, Maria Célia, Valenzuela S, Sandra Verónica, \& Do Carmo Cruz R, Maria Lúcia. (2011). EL USO DE DROGAS EN EL PERSONAL DE ENFERMERÍA. Ciencia y enfermería, 17(2), 37-45. https://dx.doi.org/10.4067/ S0717-95532011000200005

Martins ERC, Zeitoune RCG, Francisco MTR, Spindola T, Marta CB. (2009) Concepções do trabalhador de enfermagem sobre drogas: a visibilidade dos riscos. $R$ Enferm UERJ, 17(3): 368-72

Ministerio de Sanidad de España, (2011) "Prevención del consumo de alcohol en el trabajo", Secretaria de Salud Laboral, Madrid.

Stacciarini JM, Tróccoli B. (2001) O estresse na atividade ocupacional do enfermeiro. Rev Lat Am Enfermagem; 9(2): 17-25.

Beck LM David HMSL. El abuso de drogas y el mundo del trabajo: posibilidades de actuación de la enfermera. Esc. Anna Nery Rev Enferm. 2007; 11 (4): 706-11.

Aguado Martín, José Ignacio, Bátiz Cano, Ainhoa, \& Quintana Pérez, Salomé. (2013). El estrés en personal sanitario hospitalario: estado actual. Medicina y Seguridad del Trabajo, 59(231), 259-275. https://dx.doi.org/10.4321/S0465546X2013000200006

D’Anello S, Marcano E, Guerra JC. Estrés ocupacional y satisfacción laboral en médicos del Hospital Universitario de los Andes, Mérida, Venezuela. Medula, Revista de la Facultad de Medicina Universidad de los Andes. 2000; 9:1-9.

Feldman, Lya, \& Blanco, Gisela. (2012). Una aproximación al estudio de los factores psicosociales laborales en Venezuela. Salud de los Trabajadores, 20(1), 75-92. Recuperado en 06 de enero de 2017, de http://www.scielo.org.ve/scielo. php?script=sci_arttext\&pid=S1315-01382012000100007\&lng=es\&tlng=es.

Piñeiro Fraga, María. (2013). Estrés y factores relacionados en el personal sanitario de hospitalización psiquiátrica: un estudio de prevalencia. Enfermería Global, 12(31), 125-150. Recuperado en 06 de enero de 2017, de http://scielo.isciii.es/ scielo.php?script=sci_arttext\&pid=S1695-61412013000300008\&lng=es\&t1 ng=es.

Hernández, Jorge Román. (2003). Estrés y Burnout en profesionales de la salud de los niveles primario y secundario de atención. Revista Cubana de Salud Pública, 29(2), 103-110. Recuperado en 05 de enero de 2017, de http://scielo.sld.cu/scielo. 
php?script=sci_arttext\&pid=S0864-34662003000200002\&lng=es\&tlng=es.

"Sociedad española para el estudio de la ansiedad y el estrés; S.E.A.S" (sede Web). Facultad de Psicología. Universidad Complutense de Madrid. Disponible en: www.ucm.es/info/seas/estres_lab/index.htm.

Richardsen A, Burke R. 1991. Occupational stress and job satisfaction among physicians: sex differences. Social Sciences Medical33: 1179-1187

Siegrist J, Wege N, Puhlhofer F, Wahrendorf M. (2009) A short generic measure of work stress in the era of globalization: effort-reward imbalance. Int Arch Occup Environ Health; 82 (8): 1005-13.

Karasek R, Theorell T. (1990) Healthy Work: Stress productivity and the reconstruction of working life. New York: Basic Books.

Williams S, Cooper C. 1998. Measuring occupational stress. Journal of Occupational Health Psychology 3: 306-321.

Moreno-Jiménez B, Peñaloca C. 1999. El estrés asistencial en los servicios de salud. En: Simón MA (Ed.) Manual de Psicología de la Salud. Biblioteca Nueva. Madrid.

Houston K. 1997. Stress and coping. En: Zinder CR, Ford CE (Ed). Coping with negative life events. Plenum. New York.

Lazarus R. 1991. Emotion and Adaptation. Oxford University Press. New York.

Sarafino E. 1997. Health Psychology. John Wiley. New York.

C. Climent, E. Castrillón, C. Miranda, C. Gonzalez, S. Conde y F. Torres. (2015) URGENCIAS PSIQUIATRICAS. Guías de práctica clínicas basadas en la evidencia. Asociación Colombiana de facultades de medicina.

Moreno Coutiño, Ana; Medina Mora Icaza, Maria Elena, 2008, Tabaquismo y depresión Salud Mental, vol. 31, núm. 5, septiembre-octubre, pp. 409-415 Instituto Nacional de Psiquiatría Ramón de la Fuente Muñiz Distrito Federal, México

Goic A.(1991) Depresión enmascarada: Rostro médico de la depresión psíquica. Rev. Med. Chile 119 (3): 321-326.

American Psychiatric Association. 1994 Diagnostic and Statistical Manual of Mental Disorders (DSM-IV). 4ta ed. Washington : Americam Psychiatric.

López I. 1992 Clasificación de los trastornos mentales y comportamiento de las enfermedades. (CIE-10) Ginebra.

Josué Díaz, Lídice, Valdés Savín, Yissett, Quevedo Fonseca, Carlos, Torres Lio-coo, Vladimir, Font Darias, Ileana, \& Guidi Virues, Elizabeth. (2007). Depresión 
oculta en sujetos que laboran en condiciones complejas. Revista Cubana de Medicina Militar, 36(2) Recuperado en 05 de enero de 2017, de http://scielo.sld. cu/scielo.php?script=sci_arttext\&pid=S0138-65572007000200006\&lng=es\&tl $\mathrm{ng}=\mathrm{es}$.

Grau, Armand, Suñer, Rosa, \& García, María M.. (2005). Desgaste profesional en el personal sanitario y su relación con los factores personales y ambientales. Gaceta Sanitaria, 19(6), 463-470. Recuperado en 06 de enero de 2017, de http:// scielo.isciii.es/scielo.php?script=sci_arttex\&pid=S0213-91112005000600007\&$\mathrm{ng}=\mathrm{es} \&$ tlng $=\mathrm{es}$.

Vargas, Divane de, \& Dias, Ana Paula Vieira. (2011). Prevalencia de depresión en trabajadores de enfermería de Unidades de Terapia Intensiva: estudio en hospitales de una ciudad del noroeste del Estado de Sao Paulo. Revista Latino-Americana de Enfermagem, 19(5), 1114-1121. https://dx.doi.org/10.1590/ S0104-11692011000500008

García de Alba García, J E; Castañeda Aguilera, E; (2011). Depresión en el personal sanitario femenino y el análisis de los factores de riesgos sociodemográficos y laborales. Revista Colombiana de Psiquiatría, 40() 420-432. Recuperado de http:// www.redalyc.org/articulo.oa?id=80622315004

Calderón G. Depresión. Causas, manifestaciones y tratamiento. México: Trillas; 1984. p. 73-85.

Mercado J, Padilla S, Luna F. 1988, Frecuencia y factores de riesgo de trastornos emocionales en una población de consulta externa. Psiquiatría,3:219-23

Ministerio de salud 2013. DEPRESION EN PERSONAS DE 15 AÑOS A MAS. Santiago, MINSAL.

Ayuso Murillo D, Colomer Sánchez A, Herrera Peco I. 2016, Ansiedad rasgo y ansiedad estado en enfermeras gestoras y clínicas de la Comunidad de Madrid. Metas Enferm abr 2016, Madrid.

Piqueras, J.A., Martínez, A.E, Ramos, V, Rivero, R; García, L.J., Oblitas, L.A. (2008). Ansiedad, Depresión y Salud, Suma Psicológica, 5(10), 43-74

Sierra, J.C., Ortega, V. \& Zubeidat, I (2003). Ansiedad, angustia y estrés: tres conceptos a diferenciar, Revista mal-estar e subjetividade/ fortaleza, 3(1), pp. 10-59

Hernández, J.R. (2003). Estrés y Burnout en profesionales de la salud de los niveles primario y secundario de atención. Revista Cubana Salud Pública [versión electrónica], 29 (2), 103-110.

Muiños Martínez, R; García Galindo, J T; Ferrer-García, M; Tous Ral, J M; (2012). La ansiedad como estigma: el estereotipo de la persona ansiosa en la población clínica, sanitaria y general. Anuario de Psicología, 42() 113-127. 
Recuperado de http://www.redalyc.org/articulo.oa?id=97024524008

Virues, R. A. (2005). Estudio sobre ansiedad. Revista PsicologiaCientifica.com, 7(8).

Disponible en: http://www.psicologiacientifica.com/ansiedad-estudio 